\title{
A Freeness and Sharing Analysis of Logic Programs Based on a Pre-interpretation
}

\author{
Maurice Bruynooghe ${ }^{1}$, Bart Demoen ${ }^{1}$, Dmitri Boulanger ${ }^{2}$, Marc Denecker $^{1}$ and \\ Anne Mulkers ${ }^{1}$ \\ 1 K.U.Leuven, Department of Computer Science, \\ Celestijnenlaan 200A, 3001 Heverlee, Belgium \\ email: $\left\{\right.$ maurice, bimbart,marcd,anne\}@cs.kuleuven.ac.be ${ }^{\star \star \star}$ \\ ${ }^{2}$ CNUCE Istituto del CNR, via S.Maria,36, 56126, Pisa, Italy \\ email: dima@orione.cnuce.cnr.it ${ }^{\dagger}$
}

\begin{abstract}
Recently, a technique was presented for using pre-interpretations for abstract interpretation of the correct answer set $\mathcal{C}_{P}$ of a logic program $P$. So far it was an open problem whether pre-interpretations could also be used for deriving non-downward closed properties of the computed answer set $\mathcal{O}_{P}$ such as definite freeness. This paper shows that it is possible to do so.
\end{abstract}

\section{Introduction}

Cousot and Cousot [13, 14] have developed a widely applicable framework for abstract interpretation and obtained the following basic result: if an abstract operator $\mu^{\mathcal{A}}$ over an abstract domain $D o m^{\mathcal{A}}$ of data descriptions $\gamma$-approximates a corresponding concrete operator $\mu^{\mathcal{C}}$ over a concrete domain Dom ${ }^{\mathcal{C}}$ of data elements, and both operators are monotonic functions, then the fixpoints of both operators are such that $l f p\left(\mu^{\mathcal{A}}\right) \gamma$-approximates $l f p\left(\mu^{\mathcal{C}}\right)$.

For logic programs, the concrete operator $\mu^{\mathcal{C}}$ can be a bottom-up semantic function or a top-down one. In both cases, the execution essentially consists of sequences of unifications and projections. The latter ensure that the involved substitutions have finite domains. The construction of the abstract operator $\mu^{\mathcal{A}}$ involves replacing unification and projection by corresponding abstract operations. These abstract operations then can be plugged into the bottom-up or top-down frameworks which compute the fixpoint of the abstract operator $\mu^{\mathcal{A}}$.

A pre-interpretation $J$ of a language is a model semantics concept [20], which can be extended to map a tuple of terms $\bar{t}$ to $J(\bar{t})$, a set of tuples of domain elements, a so called domain relation. The domain relation $J(\bar{t})$ encodes properties

\footnotetext{
${ }^{\star \star \star}$ Research supported by the Flemish section of "National Fund for Scientific Research", GOA "Non-standard applications of abstract interpretation", project IT/IF/04, project ISC-IL-90-PARFORCE, HCM project CHRX-CT94-0624, INTAS project 93-1702.

$\dagger$ Research supported by the ERCIM Grant No.95-01. A part of this work has been done at the GMD FIRST, Berlin.
} 
of the tuple. In particular, the pre-interpretation used in this paper expresses freeness, groundness and sharing properties.

Following work of Giacobazzi et al [17] on a generalised algebraic semantics for constraint logic programs, $[2,1]$ introduced pre-interpretations based abstractions of constraint computations and used them to approximate the $s$-semantics of logic programs. However, a safe abstraction of non-downward closed properties of computed answers was only ensured when execution of the program did not require the so called "peel"-rule of the Martelli-Montanari unification algorithm [21] and the papers did not offer algorithms for checking the latter. The ideas led Gallagher et al. [16] to use pre-interpretation based models for the derivation of a wide range of declarative properties (for declarative properties, the above safety problem disappears). Their experiments showed that this is an approach with great practical value. Also the compiled approach of [8] can be understood as computing a model of the program under some pre-interpretation.

We return to the problem of pre-interpretations based analysis for nondownward closed properties of computed answers. Our abstract domain is the powerset of the original one. The paper explains how familiar properties can be extracted from elements in this abstract domain and develops a 3 element pre-interpretation (gif). It gives an algorithm for abstracting unification and extends the notion of "peel-free" unification. It compares the domain with the well-known Share+Free domain $[18,23]$ and reports on a first implementation. Our analysis not only allows to derive definite freeness and possible sharing but also to derive definite sharing. The latter information is useful for the ANDparallelisation of logic programs. It allows to reduce independence conditions to false and to identify parts of the program which are inherently sequential [7].

Our analysis is not obtained just by the systematic lifting to the powerset of the original domain as studied e.g. in [15]. That approach cannot lead to the derivation of non-downward closed properties.

Section 2 recalls some preliminaries and explains the use of tuples. Section 3 recalls what a pre-interpretation is and introduces domain relations. Section 4 introduces the gif abstract domain and section 5 explains how to extract properties from an element in this abstract domain. The abstract operations are introduced in section 6. Section 7 makes a comparison with the Share + Free domain and reports on an implementation.

\section{Preliminaries}

Terms in a language are syntactic objects built from a finite set of functors and a enumerable set of variables. With $o$ denoting an object, $\bar{o}$ denotes a tuple of objects. $\operatorname{Var}(o)$ denotes the set of variables in a syntactic object $o$. Variables are denoted by capital letters $X, Y, \ldots$. Abusing notation, we use $\bar{X}$ not only to denote a tuple of variables, but in some contexts also to denote the corresponding set of variables. $\overline{\boldsymbol{o}}_{1} \cdot \overline{\boldsymbol{o}}_{2}$ denotes the tuple which is the concatenation of the tuples $\overline{\boldsymbol{o}}_{1}$ and $\overline{\boldsymbol{o}}_{2}$. A tuple constructed from $\overline{\boldsymbol{o}}_{1}$ and $\overline{\boldsymbol{o}}_{2}$ but with elimination of duplicates is denoted $\bar{o}_{1} \cup \bar{o}_{2}$. A substitution is a function mapping a syntactic object to a 
syntactic object by simultaneously replacing a finite set of variables by terms. They are denoted by $\sigma, \theta, \ldots$. We write $t \theta$ for the application of a substitution to a term. $\operatorname{Dom}(\theta)$ denotes the domain of the substitution $\theta$, i.e. the variables which are replaced by terms. An equation system $E$ is a finite set of equations between terms. Abusing notation, we write $\{\bar{s}=\bar{t}\}$ for the equation system $\left\{s_{1}=\right.$ $\left.t_{1}, \ldots, s_{n}=t_{n}\right\}$. In the well-known solved form as computed by the MartelliMontanari unification algorithm [21], left-hand sides are distinct variables which do not occur in the right-hand sides. We make also use of a pre-solved form which is a system of equations which is in solved form after removal of all equations of the form $X=X$. We fix a partial function $m g u(E)$ mapping an equation system $E$ to a most general unifier. With $\theta$ a substitution, eqn $(\theta)$ denotes the equations system $\{X=X \theta \mid X \in \operatorname{Dom}(\theta)\}$.

As stated in the introduction, an analysis is constructed by replacing primitive operations over concrete data in an appropriate concrete semantics by abstract operations over data descriptions. For logic programs, the relevant data in the concrete operational semantics are usually considered to be (accumulated) substitutions. In our setting, it is more convenient to consider pairs $\bar{X}, \bar{t}$ where $\bar{X}$ is a so called reference domain, a set of so called reference variables and $\bar{t}$ is a tuple of terms such that $\bar{X}=\bar{t}$ is in pre-solved form. For example, where usually the computed answer is defined as $\sigma \uparrow \bar{X}$, the restriction of the accumulated substitution $\sigma$ to the variables $\bar{X}$ of the query, we say $\bar{X}$ is the reference domain and $\bar{X} \sigma$ is the computed answer. There is an obvious one to one correspondence between pairs $\bar{X}, \bar{t}$ such that $\bar{X}=\bar{t}$ is in pre-solved form and idempotent substitutions $\sigma$ with $\operatorname{Dom}(\sigma) \subseteq \bar{X}$ : the tuple corresponding to $\sigma$ is $\bar{X} \sigma$ and the substitution corresponding to $\bar{t}$ is $m g u(\{\bar{X}=\bar{t}\})=\left\{X / \bar{t}_{X} \mid \bar{t}_{X} \neq X\right\}$. Variable names inside tuples are irrelevant to the extent that the tuple remains in pre-solved form wrt. the reference domain. This corresponds to the irrelevance of variable names of the clause variants used in $S L D$-derivations, to the extent that they do not conflict with variable names already in use.

\section{Pre-interpretations}

Following [20], a pre-interpretation $J$ for a language $\mathcal{L}$ is characterised by a domain $D_{J}$ and, for each $n$-ary functor $f / n$ of the language, a function $f_{J}$ mapping elements of $D_{J}^{n}$ to $D_{J}$. A variable assignment $V$ associates an element of $D_{J}$ with each variable in $\mathcal{L}$. Let $V(o)$ denote the syntactic object obtained by replacing the variables of $o$ by the domain elements specified by $V$. We can define a function $\tilde{J}$ which maps a term without variables but with domain elements of $D_{J}$ to a domain element: $\tilde{J}\left(f\left(t_{1}, \ldots, t_{n}\right)\right)=f_{J}\left(\tilde{J}\left(t_{1}\right), \ldots, \tilde{J}\left(t_{n}\right)\right)$ for $f / n \in \mathcal{L}$ and $\tilde{J}(d)=d$ for $d \in D_{J}$.

Using this mapping, we can extend $J$ into a mapping from a tuple of nonground terms to a domain relation, called the pre-interpretation of the tuple: $J\left(\left\langle t_{1}, \ldots, t_{n}\right\rangle\right)=\left\{\left\langle\tilde{J}\left(V\left(t_{1}\right)\right), \ldots, \tilde{J}\left(V\left(t_{n}\right)\right)\right\rangle \mid V\right.$ is a variable assignment $\}$. Notice that tuples which are renamings of each other have the same pre-interpretation. Another consequence of this definition is the following trivial proposition: 
Proposition 1. $\bar{t}_{1} \leq \bar{t}_{2}$ implies $J\left(\bar{t}_{1}\right) \subseteq J\left(\bar{t}_{2}\right)$.

The pre-interpretation on which the freeness and sharing analysis in this paper is based is the gif pre-interpretation $J^{g i f}$. It is based on a three element domain $D=\{g, i, f\}$. The pre-interpretation of a functor $f / n$ is defined as: if $\forall j: 1 \leq j \leq n: d_{j}=g$ then $f_{J g i f}\left(d_{1}, \ldots, d_{n}\right)=g$ else $i$. It gives distinct domain relations for free variables, ground terms and partially instantiated terms. The domain element $f$ is not in the range of the pre-interpretation of any functor, it is called a non-term element $[2,1]$.

Example 1. $J^{g i f}(\langle X\rangle)=\{\langle f\rangle,\langle i\rangle,\langle g\rangle\}$.

$J^{g i f}(\langle X, X\rangle)=\{\langle f, f\rangle,\langle i, i\rangle,\langle g, g\rangle\}$.

$J^{g i f}(\langle X, Y\rangle)=\{\langle f, f\rangle,\langle f, i\rangle,\langle f, g\rangle,\langle i, f\rangle,\langle i, i\rangle,\langle i, g\rangle,\langle g, f\rangle,\langle g, i\rangle,\langle g, g\rangle\}$.

$J^{g i f}(\langle f(Y), f(Z)\rangle)=J^{g i f}(\langle f(X, Y), f(X, Z)\rangle)=\{\langle i, i\rangle,\langle i, g\rangle,\langle g, i\rangle,\langle g, g\rangle\}$.

$J^{g i f}(\langle f(X), f(X)\rangle)=\{\langle i, i\rangle,\langle g, g\rangle\}$.

\section{Abstract Domain based on a pre-interpretation $J$}

Besides the notation $\bar{t}$ for a n-tuple of terms, we need several others. We use $\bar{T}$ for a set of n-tuples of terms and $\overline{\mathcal{T}}$ for the set of all n-tuples. Finally, $\mathcal{P}(\overline{\mathcal{T}})$ is the powerset of all n-tuples. For domain tuples, we need even more. We use $\bar{d}$ for a n-tuple of domain elements from some domain $D$, and $D R$ for a set of domain tuples (a Domain Relation), which is a subset of $D^{n}$, the set of all domain tuples. Finally, we use $S D R$ for a Set of Domain Relations, $S \mathcal{L} \mathcal{R}$ for the set of all domain relations and $\mathcal{P}(\mathcal{S D R})$ for the powerset of $S D R$.

The reference domain $\bar{X}$ associated with a tuple of terms $\bar{t}$ or domain elements $\bar{d}$ can be considered as attributes. To select the tuple consisting of components from a subset $\bar{Y}$ of the attributes, we use the notations $\bar{t}_{\bar{Y}}$ and $\bar{d}_{\bar{Y}}$ (and $\bar{t}_{Y}$ if a single attribute is selected).

The establishment of an abstract domain and a Galois connection between a concrete and abstract domain is a first step towards a program analysis. We extend the pre-interpretation as introduced in section 3 into an abstraction function from a set of tuples into a set of domain relations and obtain a Galois connection.

Proposition 2. Let $\alpha: \mathcal{P}(\overline{\mathcal{T}}) \rightarrow \mathcal{P}(\mathcal{S D R})$ be defined as $\alpha(\bar{T})=\{J(\bar{t}) \mid \bar{t} \in \bar{T}\}$. Let $\gamma: \mathcal{P}(\mathcal{S D R}) \rightarrow \mathcal{P}(\overline{\mathcal{T}})$ be defined as $\gamma(S D R)=\{\bar{t} \mid J(\bar{t}) \in S D R\}$.

Then the quadruple $((\mathcal{P}(\overline{\mathcal{T}}), \subseteq), \alpha,(\mathcal{P}(\mathcal{S D} \mathcal{R}), \subseteq), \gamma)$ is a Galois connection.

Notice that this is not a Galois insertion; $\mathcal{P}(\mathcal{S D R})$ has spurious elements, due to the existence of non empty domain relations which cannot be the preinterpretation of any tuple, e.g $\{\langle f\rangle,\langle g\rangle\}$.

Pre-interpretation based analyses or more precisely, model based analyses so far $[16,3]$ use the standard notion of a model to approximate the set of correct answers of a program. A model only provides approximations describing downward closed sets of tuples. Therefore, only downward closed properties can be derived. 


\section{Decoding an element of the abstract domain}

Given a tuple of terms $\bar{t}$ with reference domain $\bar{X}$, we can formulate predicates and one function (sharegroups) expressing mostly familiar [5] properties of a tuple of terms ${ }^{5}: \operatorname{free}(X, \bar{t})$ iff $\bar{t}_{X}$ is a variable; $\operatorname{ground}(X, \bar{t})$ iff $\bar{t}_{X}$ is ground; instantiated $(X, \bar{t})$ iff $\bar{t}_{X}$ is instantiated but not ground; sharegroup $(\bar{Y}, \bar{t})$ iff $\exists U$ s.t. $\bar{Y}=\left\{Y \in \bar{X} \mid U \in \operatorname{Var}\left(\bar{t}_{Y}\right)\right\} ;$ sharegroups $(\bar{t})=\{\bar{Y} \mid$ sharegroup $(\bar{Y}, \bar{t})\}$; indep $(\bar{Y}, \bar{Z}, \bar{t})$ iff $\operatorname{Var}\left(\bar{t}_{\bar{Y}}\right) \cap \operatorname{Var}\left(\bar{t}_{\bar{Z}}\right)=\emptyset$. They can be lifted to sets expressing either a $\mathcal{D}$ efinite property or a $\mathcal{P}$ ossible property. For example: $\mathcal{D} \operatorname{sharegroup}(\bar{Y}, \bar{T})$ iff $\forall \bar{t} \in \bar{T}:$ sharegroup $(\bar{Y}, \bar{t}) ; \mathcal{P}$ sharegroup $(\bar{Y}, \bar{T})$ iff $\exists \bar{t} \in \bar{T}: \operatorname{sharegroup}(\bar{Y}, \bar{t})$. They can be further extended towards domain relations. For example:

$\mathcal{P}$ sharegroups $(D R)=\mathcal{P}$ sharegroups $(\bar{T})$ where $\bar{T}=\{\bar{t} \mid D R=J(\bar{t})\}$. We need constructive definitions for the properties of interest. Straightforward ones are ${ }^{6}$ :

Proposition 3. Let $D R$ be a domain relation in $J^{g i f}$, and $X$ a reference variable in reference domain $\bar{X}$.

$\mathcal{D}$ free $(X, D R)$ iff $\mathcal{P}$ free $(X, D R)$ iff $\exists \bar{d} \in D R: \bar{d}_{X}=f$.

$\mathcal{D}$ ground $(X, D R)$ iff $\mathcal{P}$ ground $(X, D R)$ iff $\forall \bar{d} \in D R: \bar{d}_{X}=g$.

$\mathcal{D}$ instantiated $(X, D R)$ iff $\neg \mathcal{D}$ free $(X, D R)$ and $\neg \mathcal{D}$ ground $(X, D R)$.

How to compute $\mathcal{D}$ sharegroups $(D R)$ and $\mathcal{P}$ sharegroups $(D R)$ is less obvious. We define $\operatorname{Pos}(\bar{Y}, \bar{X})=\bar{d}$ where $\bar{d}_{\bar{Y}}=\bar{i}_{i}$ and $\bar{d}_{\bar{X} \backslash \bar{Y}}=\bar{g}$ and look at an example.

Example 2. For a reference domain $\langle X, Y, Z\rangle$, consider the domain relation $D R=\{\langle g, g, g\rangle,\langle i, g, g\rangle,\langle i, i, g\rangle,\langle g, g, i\rangle,\langle i, g, i\rangle,\langle i, i, i\rangle\}$. Typical tuples are $\bar{t}_{1}=$ $\langle f(U, V), f(V), f(W)\rangle$ and $\bar{t}_{2}=\langle f(U, V, S, T), f(V, S), f(W, S, T)\rangle$. The two tuples have sharegroups $\{X\},\{X, Y\},\{Z\}$, but $\bar{t}_{2}$ has additional sharegroups $\{X, Y, Z\}$ and $\{X, Z\}$. Careful investigation shows that all tuples described by $D R$ share this characteristic, i.e. D sharegroups $(D R)=\{\{X\},\{X, Y\},\{Z\}\}$ and $\mathcal{P}$ sharegroups $(D R)=\mathcal{D}$ sharegroups $(D R) \cup\{\{X, Z\},\{X, Y, Z\}\}$. The application of $\mathcal{P}$ os on the sharegroups yields elements of $D R$ e.g. $\mathcal{P} o s(\langle X, Y\rangle,\langle X, Y, Z\rangle)$ $=\langle i, i, g\rangle \in D R$. Also, non-definite possible sharegroups (e.g. $\{X, Y, Z\})$ can be obtained as the union of strictly smaller sharegroups, while definite sharegroups cannot.

For the definition below, we use the order $g<i<f$. We call an element $\bar{d} \in D R$ primitive iff $\bar{d}$ is not the arguments-wise maximum of a subset $S \subseteq D R$ which does not contain $\bar{d}$. Formally, with $\bar{X}$ the reference domain of $D R$ :

$\operatorname{Primitive}(\bar{Y}, D R)$ iff $\mathcal{P}$ os $(\bar{Y}, \bar{X}) \in D R$ and

$\forall S: S \subseteq(D R \backslash\{\mathcal{P}$ os $(\bar{Y}, \bar{X})\})$ implies $\operatorname{Pos}(\bar{Y}, \bar{X}) \neq \max (S)$

where $\max \left(\left\{\bar{d}, \bar{d}^{\prime}, \bar{d}^{\prime \prime}, \ldots\right\}\right)_{Y}=\max \left(\left\{\bar{d}_{Y}, \bar{d}_{Y}^{\prime}, \bar{d}_{Y}^{\prime \prime}, \ldots\right\}\right)$.

The proposition below states that for $J^{\text {gif }}$ the primitive sharegroups are the definite sharegroups. Candidate possible sharegroups are the sharegroups

\footnotetext{
${ }^{5}$ A more complete set is in the full version of the paper [6].

${ }^{6}$ Notice that $J^{\text {gif }}$ provides a strong encoding of freeness, groundness and instantiatedness.
} 
$\bar{Y}$ such that $\operatorname{Pos}(\bar{Y}, \bar{X}) \in D R$. However, in case freeness occurs, some of these candidates can be rejected, as shown in the following example.

Example 3. For a reference domain $\langle X, Y\rangle$, consider the domain relation $D R=$ $\{\langle g, g\rangle,\langle i, g\rangle,\langle f, g\rangle,\langle g, i\rangle,\langle i, i\rangle,\langle f, i\rangle\}$. D sharegroups $(D R)=\{\{X\},\{Y\}\}$. Moreover, for any $\bar{t}$ such that $J(\bar{t})=D R, \bar{t}_{X}$ is free and does not occur in $\bar{t}_{Y}$, otherwise $\langle f, g\rangle$ would not be an element of $D R$. Hence, there cannot be sharing between $X$ and $Y$. So here is a case in which $\mathcal{P}$ sharegroups $(D R)=\mathcal{D}$ sharegroups $(D R)$.

Proposition 4. Let $D R$ be a domain relation in $J^{g i f}$ with reference domain $\bar{X}$. Let $\bar{Y} \subseteq \bar{X}$ such that $\mathcal{P}$ os $(\bar{Y}, \bar{X}) \in D R$, then:

$\bar{Y} \in \mathcal{D}$ sharegroups $(D R)$ iff Primitive $(\bar{Y}, D R)$.

$\bar{Y} \in \mathcal{P}$ sharegroups $(D R)$ iff $(\exists Y \in \bar{Y}: \mathcal{D}$ free $(Y, D R)) \rightarrow \operatorname{Primitive}(\bar{Y}, D R)$.

Independence can be extracted through its relationship with sharegroups: indep $\left(\bar{X}_{1}, \bar{X}_{2}, \bar{t}\right)$ iff $\forall \bar{Y} \in$ sharegroups $(\bar{X}, \bar{t}): \bar{Y} \cap \bar{X}_{1}=\emptyset$ or $\bar{Y} \cap \bar{X}_{2}=\emptyset$ :

Proposition 5. Dindep $\left(\bar{X}_{1}, \bar{X}_{2}, D R\right)$ iff $\forall \bar{Y} \in \mathcal{P}$ sharegroups $(D R): \bar{Y} \cap \bar{X}_{1}=$ $\emptyset$ or $\bar{Y} \cap \bar{X}_{2}=\emptyset$. P indep $\left(\bar{X}_{1}, \bar{X}_{2}, D R\right)$ iff $\forall \bar{Y} \in \mathcal{D}$ sharegroups $(D R): \bar{Y} \cap \bar{X}_{1}=$ $\emptyset$ or $\bar{Y} \cap \bar{X}_{2}=\emptyset$.

Finally, to decode an element of the abstract domain, we extend these predicates for a set of domain relations. The extension is straightforward. Definite properties hold if they hold for each domain relation, $\mathcal{P}$ ossible properties hold if they hold for at least one domain relation. Further on, sets of domain relations which are the powerset of a single one, will be of special importance. Their concretisation gives a set of downward closed tuples of terms. For our analysis to be practical, we should have a more effective way of computing their properties of interest. One can prove:

$\mathcal{D}$ free $(X, S D R)$ iff $\forall D R \in S D R: \mathcal{D}$ free $(X, D R)$.

$\mathcal{P}$ sharegroup $(\bar{Y}, S D R)$ iff $\exists D R \in S D R: \mathcal{P}$ sharegroup $(\bar{Y}, D R)$.

$\mathcal{D}$ free $(X, \mathcal{P}(D R))=$ false.

Dground $(X, \mathcal{P}(D R))=$ Dground $(X, D R)$.

$\bar{Y} \in \mathcal{P}$ sharegroups $(\mathcal{P}(D R))$ iff $\forall X \in \bar{Y}: \neg \mathcal{D}$ ground $(X, D R)$.

Dindep $\left(\bar{X}_{1}, \bar{X}_{2}, \mathcal{P}(D R)\right)$ iff $\forall X \in \bar{X}_{1}: \mathcal{D}$ ground $(X, D R)$

or $\forall X \in \bar{X}_{2}:$ Dground $(X, D R)$.

\section{Abstract Operations}

Two key operations are unification and conjunction. In our setting, unification is a partial function, defined for $\bar{X}=\bar{t}$ a pre-solved form and $\bar{t}$ and $E$ standardised apart $^{7}:$ unif $(\bar{X}, \bar{t}, E)=(\bar{X} \cup \operatorname{Var}(E)) \sigma$ where $\sigma=m g u(\{\bar{X}=\bar{t}\} \cup E)$. Conjunction is also a partial function, defined for $\bar{X}_{1}=\bar{t}_{1}$ and $\bar{X}_{2}=\bar{t}_{2}$ pre-solved

\footnotetext{
${ }^{7}$ Apart from variables in $\bar{X}, \bar{t}$ and $E$ do not share variables.
} 
forms and $\bar{t}_{1}$ and $\bar{t}_{2}$ standardised apart ${ }^{8}: \operatorname{conj}\left(\bar{X}_{1}, \bar{t}_{1}, \bar{X}_{2}, \bar{t}_{2}\right)=\left(\bar{X}_{1} \cup \bar{X}_{2}\right) \sigma$ where $\sigma=m g u\left(\bar{X}_{1}=\bar{t}_{1} \cup \bar{X}_{2}=\bar{t}_{2}\right)$.

Because conjunction can be defined in terms of relational operators, we define abstract unification in terms of abstract conjunction ${ }^{9}$ :

$A b s t r \_u n i f(\bar{X}, S D R, E)=A b s t r \_\operatorname{conj}(\bar{X}, S D R, \operatorname{Var}(E), \alpha(\{\operatorname{Var}(E) m g u(E)\}))$

Proposition 6. If Abstr_conj $\left(\bar{X}_{1}, S D R_{1}, \bar{X}_{2}, S D R_{2}\right) \gamma$ - approximates $\operatorname{conj}\left(\bar{X}_{1}, \bar{t}_{1}, \bar{X}_{2}, \bar{t}_{2}\right)$ for $\bar{t}_{1} \in \gamma\left(S D R_{1}\right)$ and $\bar{t}_{2} \in \gamma\left(S D R_{2}\right)$ then

Abstr_unif $(\bar{X}, S D R, E) \gamma$-approximates unif $(\bar{X}, \bar{t}, E)$ for $\bar{t} \in \gamma(S D R)$.

The abstract elements are sets of domain relations. Therefore we define Abstr_conj in terms of an operator on pairs of domain relations (the relational conjunction operator Aconj): Abstr_conj $\left(\bar{X}_{1}, S D R_{1}, \bar{X}_{2}, S D R_{2}\right)=$ $\bigcup\left\{\operatorname{Aconj}\left(\bar{X}_{1}, D R_{1}, \bar{X}_{2}, D R_{2}\right) \mid D R_{1} \in S D R_{1}, D R_{2} \in S D R_{2}\right\}$.

Proposition 7. If $A \operatorname{conj}\left(\bar{X}_{1}, D R_{1}, \bar{X}_{2}, D R_{2}\right) \gamma$-approximates $\operatorname{conj}\left(\bar{X}_{1}, \bar{t}_{1}, \bar{X}_{2}, \bar{t}_{2}\right)$ for $\bar{t}_{1} \in \gamma\left(\left\{D R_{1}\right\}\right)$ and for $\bar{t}_{2} \in \gamma\left(\left\{D R_{2}\right\}\right)$ then Abstr_conj $\left(\bar{X}_{1}, S D R_{1}, \bar{X}_{2}, S D R_{2}\right)$ $\gamma$-approximates conj $\left(\bar{X}_{1}, \bar{t}_{1}, \bar{X}_{2}, \bar{t}_{2}\right)$ for $\bar{t}_{1} \in \gamma\left(S D R_{1}\right)$ and for $\bar{t}_{2} \in \gamma\left(S D R_{2}\right)$.

How can Aconj be implemented? Let $\bar{X}_{1}=\bar{X} \cdot \bar{Y}, \bar{X}_{2}=\bar{X} \cdot \bar{Z}$ with $\bar{Y} \cap \bar{Z}=\emptyset$. For tuples $\bar{t}_{1}, \bar{t}_{2}$ standardised apart, let $\sigma=m g u\left(\bar{X}_{1}=\bar{t}_{1} \cup \bar{X}_{2}=\bar{t}_{2}\right)=$ $m g u\left(\bar{X}=\bar{t}_{1 \bar{X}} \cup \bar{X}=\bar{t}_{2} \cup \bar{Y}=\bar{t}_{1} \bar{Y} \cup \bar{Z}=\bar{t}_{2} \bar{Z}\right) \cdot(\bar{X} \cdot \bar{Y}) \sigma$ is an instance of $\bar{t}_{1}$; so, according to Proposition $1, J((\bar{X} . \bar{Y}) \sigma) \subseteq J\left(\bar{t}_{1}\right)=D R_{1}$. Note that $J((\bar{X} . \bar{Y}) \sigma)$ is the projection of $J((\bar{X} \cdot \bar{Y} \cdot \bar{Z}) \sigma)$ on $\bar{X} . \bar{Y}$. Similar, the projection of $J((\bar{X} \cdot \bar{Y} \cdot \bar{Z}) \sigma)$ on $\bar{X} \cdot \bar{Z}$ is $J((\bar{X} \cdot \bar{Z}) \sigma)$ which is a subset of $D R_{2}$. But this means that $J((\bar{X} \cdot \bar{Y} \cdot \bar{Z}) \sigma) \subseteq D R_{1} \bowtie D R_{2}$, where $\bowtie$ is the equijoin [26] of the two domain relations. So, we can define a first relation conjunction operator: $A \operatorname{conj} j_{1}\left(\bar{X}_{1}, D R_{1}, \bar{X}_{2}, D R_{2}\right)=\mathcal{P}\left(D R_{1} \bowtie D R_{2}\right)^{10}$.

Proposition 8. Aconj $j_{1}\left(\bar{X}_{1}, D R_{1}, \bar{X}_{2}, D R_{2}\right) \gamma$-approximates $\operatorname{conj}\left(\bar{X}_{1}, \bar{t}_{1}, \bar{X}_{2}, \bar{t}_{2}\right)$ for all $\bar{t}_{1} \in \gamma\left(\left\{D R_{1}\right\}\right)$ and $\bar{t}_{2} \in \gamma\left(\left\{D R_{2}\right\}\right)$.

The definition of $A c o n j_{1}$ is independent of a particular pre-interpretation, alas it produces abstractions describing downward closed sets. We introduce two refinements, specific for the gif pre-interpretation.

A first improvement is based on a condition under which $J((\bar{X} \cdot \bar{Y} \cdot \bar{Z}) \sigma)=$ $D R_{1} \bowtie D R_{2}$. Let $\bar{t}_{1}$ and $\bar{t}_{2}$ be standardised apart and let $\sigma=m g u\left(\bar{X}_{1}=\bar{t}_{1} \cup\right.$ $\left.\bar{X}_{2}=\bar{t}_{2}\right)$. It is straightforward that $D R_{1}=J\left(\bar{t}_{1}\right)=\left\{V\left(\bar{X}_{1}\right) \mid J, V \models \bar{X}_{1}=\bar{t}_{1}\right\}$. Analogously, $D R_{2}=J\left(\bar{t}_{2}\right)=\left\{V\left(\bar{X}_{2}\right) \mid J, V \models \bar{X}_{2}=\bar{t}_{2}\right\}$. It follows then that $D R_{1} \bowtie D R_{2}=\left\{V(\bar{X} . \bar{Y} \cdot \bar{Z}) \mid J, V \models \bar{X}_{1}=\bar{t}_{1} \wedge \bar{X}_{2}=\bar{t}_{2}\right\}$.

The Martelli-Montanari unification algorithm produces a sequence of equation systems $E_{0}=\left(\bar{X}_{1}=\bar{t}_{1} \cup \bar{X}_{2}=\bar{t}_{2}\right), E_{1}, . ., E_{n}=\operatorname{eqn}(\sigma)$. Now assume that

\footnotetext{
${ }^{8}$ Apart from variables in $\bar{X}_{1} \cap \bar{X}_{2}, \bar{t}_{1}$ and $\bar{t}_{2}$ do not share variables.

${ }^{9}$ unif $(\bar{X}, \bar{t}, E)=\operatorname{conj}(\bar{X}, \bar{t}, \operatorname{Var}(E), \operatorname{Var}(E) m g u(E))$.

${ }^{10}$ To see the need for the $\mathcal{P}$-operator, consider $\bar{X}_{1}=\bar{X}_{2}=X, D R_{1}=D R_{2}=\{\langle i\rangle,\langle g\rangle\}$ and $\bar{t}_{1}=f(a, U), \bar{t}_{2}=f(V, b)$.
} 
for all $1 \leq j<n$, it holds that $J \models \forall\left(E_{j} \Leftrightarrow E_{j+1}\right)$, where $\Leftrightarrow$ expresses logical equivalence i.e. that each rewrite step preserves the set of value assignments which are solutions. This implies that $D R_{1} \bowtie D R_{2}=\left\{V\left(\bar{X}_{1} \cup \bar{X}_{2}\right) \mid J, V \models\right.$ $\forall(e q n(\sigma))\}=J\left(\left(\bar{X}_{1} \cup \bar{X}_{2}\right) \sigma\right)$.

So, the question now is which rewrite rules are equivalence preserving in $J$ ? With respect to any finite $J$, the only (non-failing) rewrite rule which is not equivalence preserving is the peel-rule: the equivalence $\forall(f(\bar{X})=f(\bar{Y}) \Leftrightarrow \bar{X}=$ $\bar{Y})$ is not satisfied. This explains why e.g. $\left\{V(\langle X\rangle) \mid J^{g i f}, V \models X=f(a, U) \wedge X=\right.$ $f(W, b)\}=\{\langle i\rangle,\langle g\rangle\} \neq\left\{V(\langle X\rangle) \mid J^{g i f}, V \models X=f(a, b) \wedge W=a \wedge U=b\right\}=$ $\{\langle g\rangle\}$. However, even for the peel-rule, an infinite set of instances are satisfied in $J^{g i f}$ : one easily verifies that for any ground atom $\bar{t}, J^{g i f} \models \forall(f(\bar{X})=f(\bar{t}) \Leftrightarrow$ $\bar{X}=\bar{t}$ ). We have the following proposition:

Proposition 9 ng-peel-free rewrite step. If $E$ is reduced to $E^{\prime}$ by a remove step (removal of an equation $X=X$ ), a switch step (replacing an equation $X=t$ by $t=X$ ), a substitute step (with $X=t$ an equation, $X$ is substituted by $t$ in the other equations) or a peel step (replacement of an equation $f\left(t_{1}, \ldots, t_{n}\right)=$ $f\left(s_{1}, \ldots, s_{n}\right)$ by $\left.t_{1}=s_{1}, \ldots, t_{n}=s_{n}\right)$ with at least one of the peeled terms $a$ ground term then: $J^{\text {gif }} \models \forall\left(E \Leftrightarrow E^{\prime}\right)$.

In [6], we describe and prove a condition in terms of the domain relations which guarantees the existence of a ng-peel-free rewriting to solved form. It allows to define: $A \operatorname{con} j_{2}\left(\bar{X}_{1}, D R_{1}, \bar{X}_{2}, D R_{2}\right)=$ if the conjunction is ng-peel-free then $\left\{D R_{1} \bowtie D R_{2}\right\}$ else $A \operatorname{con} j_{1}\left(\bar{X}_{1}, D R_{1}, \bar{X}_{2}, D R_{2}\right)$.

Proposition 10. Aconj $j_{2}\left(\bar{X}_{1}, D R_{1}, \bar{X}_{2}, D R_{2}\right) \gamma$-approximates $\operatorname{conj}\left(\bar{X}_{1}, \bar{t}_{1}, \bar{X}_{2}, \bar{t}_{2}\right)$ for all $\bar{t}_{1} \in \gamma\left(\left\{D R_{1}\right\}\right)$ and $\bar{t}_{2} \in \gamma\left(\left\{D R_{2}\right\}\right)$.

To implement an analysis, one needs $A \operatorname{conj} j_{2}\left(\bar{X}_{1}, D R_{1}, \operatorname{Var}(E), \alpha(\{\operatorname{Var}(E) m g u(E)\})\right)$. It can be realised as follows:

$E=m g u(E)$

while $\exists e \in E$ s.t. the conjunction of $D R_{1}$ and $\alpha(\{\operatorname{Var}(e) m g u(e)\})$ is ng-peelfree

do remove $e$ from $E$;

$\bar{X}_{1}=\bar{X}_{1} \cup \operatorname{Var}(e)$

od;

$D R_{1}=D R_{1} \bowtie \alpha(\{\operatorname{Var}(e) m g u(e)\}) ;$

if $E=\emptyset$ then $\left\{D R_{1}\right\}$

else $A \operatorname{con} j_{1}\left(\bar{X}_{1}, D R_{1}, \operatorname{Var}(E), \alpha(\{\operatorname{Var}(E) m g u(E)\})\right)$.

The conjunction of $\alpha(\{\operatorname{Var}(e) m g u(e)\})$ and $D R$ is ng-peel-free if at least one side of $e$ is a free variable or a ground term under $D R$.

When the rewriting is not ng-peel-free, all freeness is lost, also of variables which are known to be independent of those in the intersection of the two reference domains. Exploiting independence can prevent this ${ }^{11}$. The idea is to split $\bar{X}_{1}$

${ }^{11}$ It also improves the precision of the possible sharing which can be extracted from domain relations (e.g. the fourth example in Example 1). 
in two independent sub-domains $\bar{X}_{11}, \bar{X}_{12}$ such that $\mathcal{D}$ inde $p\left(\bar{X}_{11}, \bar{X}_{12}, D R_{1}\right)$, i.e. terms assigned to variables in the two sub-domains do not share variables. As a consequence, $D R_{1}$ can be written as a product $D R_{11} \times D R_{12}$. In addition, we require that $\bar{X}_{1} \cap \bar{X}_{2} \subseteq \bar{X}_{11}$. Similarly, we split $\bar{X}_{2}$ in $\bar{X}_{21} \cdot \bar{X}_{22}$ and $D R_{2}$ in $D R_{21} \times D R_{22}$ such that $\bar{X}_{1} \cap \bar{X}_{2} \subseteq \bar{X}_{21}$. Let $\bar{t}_{1}=\bar{t}_{11} \cdot \bar{t}_{12}$ and $\bar{t}_{2}=\bar{t}_{21} \cdot \bar{t}_{22}$ be standardised apart. One can state:

$m g u\left(\bar{X}_{11}=\bar{t}_{11} \cup \bar{X}_{12}=\bar{t}_{12} \cup \bar{X}_{21}=\bar{t}_{21} \cup \bar{X}_{22}=\bar{t}_{22}\right)=m g u\left(\bar{X}_{11}=\bar{t}_{11} \cup \bar{X}_{21}=\right.$ $\left.\bar{t}_{21}\right) \bigcup\left\{\bar{X}_{12} / \bar{t}_{12}, \bar{X}_{22} / \bar{t}_{22}\right\}$.

We have that $J\left(\left(\bar{X}_{11} \cdot \bar{X}_{21}\right) \sigma\right) \in \mathcal{P}\left(D R_{11} \bowtie D R_{12}\right)$ where $\sigma=m g u\left(\bar{X}_{11}=\right.$ $\left.\bar{t}_{11} \cup \bar{X}_{21}=\bar{t}_{21}\right), J\left(\bar{t}_{12}\right)=D R_{12}$, and $J\left(\bar{t}_{22}\right)=D R_{22}$.

So, we define another operator Aconj $j_{3}$ : determine maximal sub-domains $\bar{X}_{12}, \bar{X}_{22}$ such that $\bar{X}_{1}=\bar{X}_{11} \cdot \bar{X}_{12}, \bar{X}_{2}=\bar{X}_{21} \cdot \bar{X}_{22}, \bar{X}_{1} \cap \bar{X}_{2} \subseteq \bar{X}_{11} \cap \bar{X}_{21}$ and $\mathcal{D}$ indep $\left(\bar{X}_{11}, \bar{X}_{12}, D R_{1}\right)$ and $\mathcal{D}$ indep $\left(\bar{X}_{21}, \bar{X}_{22}, D R_{2}\right)$; then define: $A \operatorname{conj} j_{3}\left(\bar{X}_{1}, D R_{1}, \bar{X}_{2}, D R_{2}\right)=\mathcal{P}\left(D R_{1} \bar{X}_{11} \bowtie D R_{2} \bar{X}_{21}\right) \times D R_{1} \bar{X}_{12} \times D R_{2} \bar{X}_{22}$. $A$ conj $j_{3}$ preserves definite freeness of variables in $\bar{X}_{12}$ and in $\bar{X}_{22}{ }^{12}$. The following proposition holds:

Proposition 11. Aconj $j_{3}\left(\bar{X}_{1}, D R_{1}, \bar{X}_{2}, D R_{2}\right) \gamma$-approximates $\operatorname{conj}\left(\bar{X}_{1}, \bar{t}_{1}, \bar{X}_{2}, \bar{t}_{2}\right)$ for all $\bar{t}_{1} \in \gamma\left(\left\{D R_{1}\right\}\right)$ and $\bar{t}_{2} \in \gamma\left(\left\{D R_{2}\right\}\right)$.

To combine both improvements, $A$ conj $j_{3}$ replaces $A$ conj $j_{1}$ in the definition of Aconj $j_{2}$.

\section{Evaluation of the gif domain}

A logic program analysis using sets of possible sharegroups (the Share domain) has been introduced in [18]. In [23] it has been extended with a component expressing definite freeness (the Share + Free domain) and has further been refined with a linearity component $[4,22]$ (the Share+Free+Linear domain). All these domains express definite groundness and possible sharegroups, the latter two also definite freeness. As explained in Section 5, definite freeness and possible sharegroups can also be extracted from abstractions in our domain. We present a preliminary investigation about precision and performance of the gif domain.

\subsection{Definite groundness}

With respect to definite groundness, the gif domain is as precise as the analysis based on the $\{g, i\}$-pre-interpretation ${ }^{13}$, which is known to be equivalent with the Prop-domain which is in turn more precise than Share [12]. So, wrt. definite

12 A domain relation can express independence between a free variable and other variables. The result of $A c o n j_{3}$ is a product of three independent relations. Keeping the result as this product can maintain more independence than what is expressed by the domain relation which is the result of evaluating the product.

${ }^{13}$ Functors are interpreted in $g i$ as in gif, the only difference is the absence of the non-term element $f$. 
groundness, our analysis is more precise than Share. A formal comparison with Share + Free and Share + Free + Linear is not available ${ }^{14}$. As the precision of Prop is based on the very precise covering information, it is unlikely that there are cases where Share + Free + Linear can obtain better groundness information than Prop and the gif domain ${ }^{15}$. So, while in theory gif is more precise, in practice, we expect that both domains derive the same groundness.

\subsection{Definite Freeness}

Let us consider a situation with abstract state $a_{i n}$, and an equation $E$ over the reference domain of the form $X=t\left[Y_{1}, \ldots, Y_{n}\right]$ with $Y_{1}, \ldots, Y_{n}$ variables in the term $t$. Abstract unification derives a new abstract state $a_{\text {out }}$. We analyse cases where freeness, present in $a_{i n}$, is preserved in $a_{\text {out }}$ :

- the equation is $X=Y, X$ and $Y$ are free in $a_{i n}$. Share + Free preserves all freeness. In the gif domain, the equation is abstracted as $\{\langle f, f\rangle,\langle i, i\rangle,\langle g, g\rangle\}$, the subsequent conjunction is ng-peel-free and the equijoin preserves all freeness in $a_{i n}$.

- The equation is $X=t\left[Y_{1}, \ldots, Y_{n}\right], X$ is free in $a_{i n}$. Share + Free preserves all freeness of variables $Z$ independent of $X^{16}$. In the gif domain, the conjunction is again ng-peel-free $\left(X\right.$ is free in $\left.a_{i n}\right)$. Also here, the equijoin preserves freeness of all variables which are independent of $X$ (free variables are either equal to, or independent of $X$, this is expressed in $a_{i n}$ ).

- The equation is of the form $X=t\left[Y_{1}, \ldots, Y_{n}\right]$, neither $X$ nor $t\left[Y_{1}, \ldots, Y_{n}\right]$ is a free variable in $a_{i n}$. Share + Free preserves freeness of variables which are independent of $X, Y_{1}, \ldots, Y_{n}$. The independence optimisation $A c o n j_{3}$ in the gif domain also preserves freeness of those variables.

This suggests that both domains have the same precision wrt. definite freeness. So far, we have been unable to construct an example where Share+Free derives more freeness.

\subsection{Possible Sharegroups}

Here the comparison is not that simple. The interesting case is where the equation is of the form $X=t\left[Y_{1}, \ldots, Y_{n}\right]$ where both sides are neither free nor ground in $a_{i n}$. In this case, Share (and Share+Free) performs a kind of closure under union of all sharegroups containing one of $X, Y_{1}, \ldots, Y_{n}$. By taking the $\mathcal{P}\left(D R_{1} \bowtie D R_{2}\right)$, where $D R_{1}$ is the part of $a_{i n}$ dependent on the variables

$\overline{14}$ We are not aware of examples of pure programs where more precise groundness is obtained than with Share.

15 A typical example where Share, Share + Free and Share + Free + Linear are less precise than Prop [12] is: $p(U, U, V) . p(U, V, U) . q(U, V, W):-p(U, V, W), U=a, V=W$. However such cases rarely occur in real programs.

16 Typically, also the $Y_{i}$ are independent from $X$. 
$X, Y_{1}, \ldots, Y_{n}$ and $D R_{1}$ of the equation, the gif domain performs a somewhat similar closure operation on sharegroups.

Concerning the relative precision of the three share domains, Share + Free takes the "closure" in less cases than Share, which gives rise to more precise possible sharing. Share + Free + Linear avoids the "closure" in even more cases, though benchmarks show that this not so often further enhances precision ${ }^{17}$.

As said above, the gif domain sometimes gives better groundness. Because of the better groundness, the same example mentioned above gives also a better possible sharing than in Share+Free+Linear. However, also in other cases, the sharing can be better. Consider the similar example

$p(U, U, V) . p(U, V, U) . q(U, V, W):-p(U, V, W), V=W$. In the gif domain, we obtain $\{U, V, W\}$ as single sharegroup for $q(U, V, W)$. In Share + Free + Linear, one derives $\{U, V, W\}$ and $\{V, W\}$.

On the other hand, as Example 1 showed, the gif domain is weak at capturing independence between instantiated terms. This can cause a loss of precision. Consider: $q(X, Y, Z):-X=f(A), Y=f(A, B), Z=f(B)$. Share yields sharegroups $\{X, Y\}$ and $\{Y, Z\}$. The gif domain also derives $\{X, Y, Z\}$ although all steps are ng-peel-free.

\subsection{Implementation}

A first implementation has been realised by adapting the bottom-up Prop analyser described in [8]. The prototype implements $A C o n j_{2}$ and uses $A C o n j_{1}$ when the ng-peel-free condition is violated. The ng-peel-free check extracts all required information (e.g. which are the free and ground variables) from the domain relations according to Proposition 3 each time this information is needed. Profiling has shown that the ng-peel-free check is the most expensive part in our implementation. So, the implementation would certainly benefit from: (1) storing this information together with each domain relation and reusing it instead of recomputing it; (2) labeling at pre-compile time an equation as ng-peel-free when one side of the equation is a ground term or the first occurrence of a variable; (3) reduce the set of reference variables after the last occurrence of a variable which does not occur in the head. The powerset of a domain relation is never computed but a global flag is set, indicating that the result for all domain relations, computed from this point on, needs to be downward closed. This flag could be refined to indicate on a domain relation basis which domain relation denotes its downward closure; such a refinement might increase the precision. To assess performance and precision, we have used the gif domain in the bottomup analyser to abstract computed answers of the predicates in a standard set of benchmarks. The same analysis has been done in the Share+Free domain, using the appropriate setting of the abstract equation systems described in [22] and the top-down analysis framework AMAI described in [19]. To do this goalindependent analysis efficiently with a top-down framework, the transformation

${ }_{17}$ They are the same benchmarks for which the combination of set sharing and pair sharing [24] (which enhances set sharing with the linearity of pair sharing) shows an improved precision [11]. 
described in [10] is applied: without this transformation, the comparison would disadvantage AMAI.

The results are summarised in Table 1.

\begin{tabular}{|c|c|c|c|c|c|c|c|c|c|c|c|c|c|c|c|}
\hline \multirow[t]{2}{*}{ program } & \multicolumn{2}{|c|}{ Time } & \multirow{2}{*}{$\begin{array}{c}\text { Sh/ } \\
\text { gif }\end{array}$} & \multirow[t]{2}{*}{$\#$ pr } & \multirow{2}{*}{$\begin{array}{c}\mathrm{mx} \\
\mathrm{ar}\end{array}$} & \multicolumn{3}{|c|}{ \# diff pr } & \multirow{2}{*}{\begin{tabular}{c|}
$f g$ \\
st
\end{tabular}} & \multirow{2}{*}{\multicolumn{2}{|c|}{\begin{tabular}{|}
$\#$ fr arg \\
gif $\mid S+F$
\end{tabular}}} & \multirow{2}{*}{$\begin{array}{l}\# \mathrm{gr} \\
\arg \end{array}$} & \multirow{2}{*}{\begin{tabular}{|c|}
$\#$ \\
$\arg$ \\
\end{tabular}} & \multicolumn{2}{|c|}{ \# sh gr } \\
\hline & gif & $\mathrm{S}+\mathrm{F}$ & & & & $\mathrm{Dg} \mid \mathrm{]}$ & & Ps & & & & & & gif & $\mathrm{S}+\mathrm{F}$ \\
\hline append & 0.03 & 0.17 & 5.7 & 1 & 3 & 0 & 0 & 0 & 0 & 1 & 1 & 0 & $\begin{array}{ll}3 \\
\end{array}$ & 2 & 2 \\
\hline poyer & 2.43 & 9.45 & 3.9 & 34 & 5 & 0 & 0 & 6 & 8 & 10 & 10 & 14 & 88 & 111 & 104 \\
\hline leriv & 1.99 & 4.42 & 2.2 & 18 & 4 & 0 & 0 & 4 & 5 & 5 & 5 & 8 & 47 & 46 & 38 \\
\hline grammar & 0.06 & 0.56 & 9.3 & 6 & 3 & 0 & 0 & 0 & 1 & 4 & 4 & 4 & 17 & 9 & 9 \\
\hline peep & 11.6 & 17.1 & 1.5 & 23 & 6 & 0 & 1 & 5 & 8 & 15 & 16 & 13 & 75 & 154 & 84 \\
\hline qsort & 0.24 & 0.73 & 3.0 & 4 & 4 & 0 & 0 & 0 & 0 & 1 & 1 & 5 & 11 & 4 & 4 \\
\hline ers & 0.05 & 0.24 & 4.8 & 2 & 3 & 0 & 0 & 0 & 1 & 1 & 1 & 0 & 5 & 3 & 3 \\
\hline serialize & 0.76 & 1.19 & 1.6 & 7 & 4 & 0 & 0 & 2 & 4 & 0 & 0 & 3 & 17 & 17 & 12 \\
\hline Total & 17.16 & 33.86 & 2.0 & 95 & & 0 & 1 & 17 & 27 & 37 & 38 & 47 & 263 & 346 & 256 \\
\hline
\end{tabular}

Table 1.

Table 1 contains the name of the benchmark program, the time (in seconds) it takes to analyse the benchmark program in the gif domain and in the Share+Free domain ${ }^{18}$, the speedup of gif over Share + Free, the number of predicates in each benchmark, the maximal arity of predicates in each benchmark, the number of predicates in each benchmark for which Share + Free has more precision than gif for groundness, freeness and sharing (Share+Free has never less precision in the benchmarks), the number of predicates for which the flag indicates that only downward closed properties are derived, the number of arguments for which freeness was derived, the number of arguments for which groundness was derived (only one number, as it is the same for gif and Share +Free), the total number of argument positions, i.e. the sum of the arities of all predicates, and the number of sharing groups that were derived.

Both the bottom-up and the top-down analyser are based on precompiling a source program into another form (for the bottom-up analyser to an abstracted Prolog program, for the top-down analyser to intermediate code for an abstract interpretation machine). The time for this precompilation is not included in the timings of table 1: it is similar for both systems and small compared to the analysis time. The timing for gif does include a postprocessing step, which given the domain relations of the predicates, computes the groundness, freeness and sharing as described in section 5 : this takes about $10 \%$ of the total time for the larger benchmarks. The times are averages over 10 runs on a Sparc 20 with ProLog_by_BIM 4.0.12 .

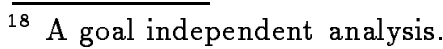


Discussion. The implementation uses a ground representation of the domain relations. This results in large domain relations for predicates with a large arity and shows up in the table as a relative slow-down for benchmarks with such predicates. We expect that a non-ground representation of the domain relations (as in [9] or [25]) would reduce the influence of large arities and on the whole benefit the implementation. However, with a rudimentary implementation of the gif domain, we arrive at a speed-up of 2 on average over AMAI.

The precision of gif in deriving groundness is exactly as for Share+Free, as expected, since situations where gif is more precise happen not to occur in the benchmarks. For freeness, gif loses the freeness of one argument (in benchmark peep). Careful analysis of why this was so, reduced it to the following example in which the freeness of $\mathrm{A}$ is lost: $t(A, Z, B):-q(B), Z=f(A, B)$. Assume q returns the powerset of $\{\langle i\rangle,\langle g\rangle\}$. The use of independence as described in Proposition 11 , cannot recover the freeness of $A$. However, doing separately the analysis for each domain relation in the powerset of $\{\langle i\rangle,\langle g\rangle\}$ recovers the freeness of $A$. These observations confirm the hypothesis that the our approach can potentially derive the same freeness as Share+Free.

On the other hand, the derived information on possible sharing is substantially weaker with gif. The source of the imprecision can be illustrated with the following example: $p(X, Y):-X=f(U), Y=f(V)$. The tuple $\langle f(U), f(V)\rangle$ is the computed answer, the abstraction cannot distinguish this from

$\langle f(U, Z), f(V, Z)\rangle$ and our current analysis derives that also $\{X, Y\}$ is a sharing group. Exploiting independence, we would derive that the result is the product of two independent domain relations $\{\langle i\rangle,\langle g\rangle\}$ and would only derive the sharegroups $\{X\}$ and $\{Y\}$, as in Share + Free.

We have also made an experimental implementation of $A c o n j_{3}$ as described in Section 6 using the independence of subsets of reference variables. We cannot yet fully report on the results of this implementation, but the following observations were made: the representations of individual domain relations is smaller but more domain relations per predicate are derived; the precision concerning freeness is the same but that concerning the possible share groups is greatly improved (no loss of precision in examples such as in the previous paragraph); the efficiency is much worse, among others, because more domain relations are derived: we currently do not know how to control this explosion.

Conclusion and future work. Our implementation of the gif-domain by means of Aconj $j_{2}$ shows that pre-interpretations are a practical means for deriving nontrivial information about logic programs without losing too much precision. More precision can be obtained by using the concept of independence, as in $A c o n j_{3}$. A first attempt to implement $A c o n j_{3}$ reduced the number of possible sharegroups drastically but resulted in a prohibitive slow-down of the analysis. We currently explore how to reduce this slow-down. Aconj $j_{3}$ is based on the idea to use the concept of independence to reduce the subset of reference variables for which the domain relation has to be be downward closed. This notion is not as powerful as one would like: the example where freeness is lost shows that not the notion of independence, but rather the notion of "being affected by a unification" is 
needed. It follows that another interesting line of research consists in exploring alternatives to $A$ conj $j_{3}$ which would improve precision and hopefully have less impact on efficiency. One possibility is to have a flag for each reference variable.

Another line of future work consists of exploring pre-interpretations that are capable of describing other interesting properties, like for example linearity, and still result in computationally well behaving analysis.

Acknowledgments. We are grateful to G. Janssens for the help with the benchmarks in the Share+Free domain and to the referees for many useful comments.

\section{References}

1. D. Boulanger and M. Bruynooghe. A systematic construction of abstract domains. In B. Le Charlier, ed., Proceedings of the first International Static Analysis Symposium, SAS'94, pp. 61-77. LNCS 864, Springer-Verlag, Sept. 1994.

2. D. Boulanger, M. Bruynooghe, and M. Denecker. Abstracting S-semantics using a model-theoretic approach. In M. Hermenegildo and J. Penjam, ed., Proceedings of the Sixth International Symposium on Programming Language Implementation and Logic Programming, PLILP'94, pp. 432-446. LNCS 844, Springer-Verlag, Sept. 1994.

3. D. Boulanger. Complete Analysis for Definite Logic Programs. Proc. $11^{\text {th }}$ Workshop Logische Programmierung, Technische Universität Wien, GMD-Studien Nr.270, September, 1995, 101-110.

4. M. Bruynooghe and M. Codish. Freeness, sharing, linearity and correctness - all at once. In P. Cousot, M. Falaschi, G. Filé, and A. Rauzy, ed., Proceedings of the Third International Workshop on Static Analysis, LNCS 724, pp. 153-164, Padova, Italy, Sept. 1993. Springer-Verlag.

5. M. Bruynooghe, M. Codish and A. Mulkers. Abstracting Unification: A key step in the design of logic program analyses. In Computer Science Today, Recent Trends and Developments, Jan van Leeuwen ed. LNCS Vol 1000, pp. 406-425, 1995. Springer-Verlag.

6. M. Bruynooghe, B. Demoen, D. Boulanger, M. Denecker and A. Mulkers. A Freeness and Sharing Analysis of Logic Programs Based on a Pre-interpretation. Report CW 233, Dept. Computer Science, KULEUVEN, May 1996.

7. F. Bueno, M. García de la Banda and M. Hermenegildo. Effectiveness of Global Analysis in Strict Independence-Based Automatic Program Parallelization. In International Logic Programming Symposium, pp320-336, 1994, MIT Press.

8. M. Codish and B. Demoen. Analysing logic programs using "prop"-ositional logic programs and a magic wand. The Journal of Logic Programming, 25(2):249-274, Dec. 1995. A preliminary version appeared in proc. ILPS'93.

9. M. Codish and B. Demoen. Deriving polymorphic type dependencies for logic programs using multiple incarnations of Prop. In B. Le Charlier, ed., Proceedings of the first International Static Analysis Symposium, SAS'94, pp. 281-296. LNCS 864, Springer-Verlag, Sept. 1994.

10. M. Codish, M. García de la Banda, M. Bruynooghe, and M. Hermenegildo. Goal dependent vs. goal independent analysis of logic programs. In Proceedings of the International Conference on Logic Programming and Automated Reasoning, LPAR'94, LNAI 822, pp. 305-319. Springer-Verlag, 1994. 
11. M. Codish, A. Mulkers, M. Bruynooghe, M. García de la Banda and M. Hermenegildo. Improving Abstract Interpretation by Combining Domains. ACM Trans. Prog.Lang. Syst. 17(1):28-44, 1995.

12. A. Cortesi, G. Filé and W. Winsborough. Optimal groundness analysis using propositional formulas. The Journal of Logic Programming, 27(2):103-179, May 1996. A preliminary version appeared in ICALP92.

13. P. Cousot and R. Cousot. Abstract interpretation: A unified lattice model for static analysis of programs by construction or approximation of fixpoints. In Proceedings of the Fourth ACM Symposium on Principles of Programming Languages, pp. 238-252, Los Angeles, 1977.

14. P. Cousot and R. Cousot. Abstract interpretation and application to logic programs. The Journal of Logic Programming, 13(2 and 3):103-179, 1992.

15. G. Filé and F. Ranzato. Improving abstract interpretations by systematic lifting to the powerset. In M. Bruynooghe, ed., Proceedings of the 1994 International Logic Programming Symposium, pp. 655-669, MIT Press.

16. J. Gallagher, D. Boulanger, and H. Saglam. Practical model-based static analysis for definite logic programs. In J. Lloyd ed., Proceedings of the 1995 International Symposium on Logic Programming, pp. 351-365, 1995, MIT Press.

17. R. Giacobazzi, S.K.Debray, and G. Levi. Generalized semantics and abstract interpretation for constraint logic programs. The Journal Logic Programming, 25(3):191-247, Dec.1995. A preliminary version appeared in Proc. FGCS92.

18. D. Jacobs and A. Langen. Static analysis of logic programs for independent ANDparallelism. The Journal of Logic Programming, 13(2 \& 3):291-314, 1992.

19. G. Janssens, M. Bruynooghe, V. Dumortier. A blueprint for an abstract machine for abstract interpretation of (constraint) logic programs. In J. Lloyd ed., Proceedings of the 1995 International Symposium on Logic Programming, pp. 336-350, 1995, MIT Press.

20. J. W. Lloyd. Foundations of Logic Programming. Springer Series : Symbolic Computation - Artificial Intelligence. Springer-Verlag, second, extended edition, 1987.

21. A. Martelli and U. Montanari. An efficient unification algorithm. ACM Transactions on Programming Languages and Systems, 4(2):258-282, Apr. 1982.

22. A. Mulkers, W. Simoens, G. Janssens, and M. Bruynooghe. On the practicality of abstract equation systems. In L. Sterling, editor, Proceedings of the International Conference on Logic Programming, pp. 781-795, Kanagawa, Japan, 1995. MIT Press.

23. K. Muthukumar and M. Hermenegildo. Combined determination of sharing and freeness of program variables through abstract interpretation. In K. Furukawa, editor, Proceedings of the Eighth International Conference on Logic Programming, pp. 49-63, Paris, France, 1991. MIT Press.

24. H. Søndergaard. An application of abstract interpretation of logic programs: Occur check reduction. In B. Robinet and R. Wilhelm, eds., ESOP'86 Proceedings European Symposium on Programming, Lecture Notes in Computer Science 213, pp. 327-338. Springer-Verlag, N.Y., 1986.

25. S. Sudarshan and R. Ramakrishnan. Optimizations of bottom-up evaluation with non-ground terms. In D. Miller, ed. Proceedings of the 1993 International Symposium on Logic Programming, pp. 557-574, 1993, MIT Press.

26. J. D. Ullman. Principles of Database Systems. Computer Science Press, 1982.

This article was processed using the $\mathrm{IAT}_{\mathrm{E}} \mathrm{X}$ macro package with LLNCS style 